\title{
Resolving Power of Algorithm for Solving the Coefficient Inverse Problem for the Geoelectric Equation
}

\author{
K. T. Iskakov and Zh. O. Oralbekova \\ L.N. Gumilyov Eurasian National University, Faculty of Information Technologies, Astana 010008, Kazakhstan \\ Correspondence should be addressed to Zh. O. Oralbekova; oralbekova@bk.ru
}

Received 9 April 2014; Revised 27 June 2014; Accepted 13 July 2014; Published 6 August 2014

Academic Editor: Valery G. Yakhno

Copyright ( 2014 K. T. Iskakov and Zh. O. Oralbekova. This is an open access article distributed under the Creative Commons Attribution License, which permits unrestricted use, distribution, and reproduction in any medium, provided the original work is properly cited.

\begin{abstract}
We considered the inverse coefficient problem for the geoelectric equation. For the purpose of research of the conditional stability of the inverse problem solution, we used integral formulation of the inverse geoelectric problem. By implementing the relevant norms and using the close system of Volterra integral equations, we managed to estimate the conditional stability of the solution of inverse problem or rather lower changes in input data imply lower changes in the solution (of the numerical method). When determining the additional information the device errors are possible. That is why this research is important for experimental studies with usage of ground penetrating radars.
\end{abstract}

\section{Introduction}

Inverse problems for hyperbolic equations, in particular for the acoustics and geoelectrics, were investigated by many authors; notably, a detailed bibliography is given in the monography of Kabanikhin [1]. We will present the main scientific results on this problem. Blagoveshchenskii applied Gelfand-Levitan method for proving the uniqueness of the solution of the inverse acoustic problem [2]. Romanov proved a comparable theorem for the following equation [3]:

$$
w_{t t}(x, t)=w_{x x}(x, t)-q(x) w(x, t),
$$

which is consolidated from the acoustic equation with wellknown transformation (see [4]):

$$
\begin{aligned}
& w(x, t)=u(x, t) \exp \left\{-\frac{1}{2} \ln \sigma(x)\right\}, \\
& q(x)=-\frac{1}{2}[\ln \sigma(x)]^{\prime \prime}+\frac{1}{4}\left[\frac{\sigma^{\prime}(x)}{\sigma(x)}\right]^{2} .
\end{aligned}
$$

Romanov and Yamamoto [5] obtained the estimation of conditional stability in $L_{2}$ for getting a multidimension analog of the inverse problem (1).
Numerical algorithm of inverse acoustic problem solving in the discrete case was given in work [6] for the first time.

Bamberger and his coauthors used a conjugate gradient method to define the acoustic impedance $[7,8]$.

$\mathrm{He}$ and Kabanikhin used the optimization method to solve the inverse problem for three-dimension acoustic equation [9].

Azamatov and Kabanikhin studied the conditional stability of the solution to Volterra operator equation in $L_{2}[10]$.

Problems of uniqueness of the inverse problem solution and set of numerical methods for solving the geoelectric equation were given in the monograph of Romanov and Kabanikhin [11].

For solving inverse acoustic problem in integral case formulation the estimation of the conditional stability in $H^{1}$ was obtained in the work of Kabanikhin et al. [12].

Further, in works $[13,14]$ for minimizing purposes they built and investigated a special form of the composite functional that allowed proving the following theorems in the space $L_{2}$ : the local correctness theorem, the correctness theorem of the inverse problem for small amount of data, and the correctness theorem in the envelope of the exact solution in $L_{2}$. 
Bukhgeim and Klibanov suggested using the method of Carleman estimates when proving uniqueness theorems of the coefficient inverse problems [15]. A broad overview on the use of Carleman estimates in the theory of multidimension coefficient inverse problems is given in the work [16].

The problem of uniqueness of inverse problem solution for determination of the coefficients of the permittivity and conductivity for Maxwell's equation system is considered in the work [17].

Approbation of the globally convergent numerical algorithm with the use of experimental radar data for determination of the permittivity is given in work [18]. They presented an analysis of convergence of the method and it has been shown that the computed and real values of permittivity were in enough agreement. A wide range of globally convergent algorithms of solving a class of problems is described in work [19].

Comparative analysis of the classical equation methods and globally convergent numerical method of solving the coefficient inverse problems was given in work [20]. These comparisons were performed for both computationally simulated and experimental data.

In the work [21] continuation problem from the timelike surface for the 2D Maxwell's equation was considered. The gradient method for the continuation and coefficient inverse problem was explained. The results of computational experiment were presented.

In this research, following the methods which were described in the work [12], we obtained the estimation results of the conditional stability of the geoelectric equation in $H^{1}$.

Herein after the second paragraph there is the conclusion of the main equations which were derived from the system of Maxwell's equations [11].

In the third paragraph we had amplified the inverse problem for the geoelectric equation with data on characteristics. It allows us to obtain a close system of integral equations.

Finally, in the fourth paragraph, the implementation of the relevant class of input data functions and the class of solutions of the inverse problem allowed us to estimate the conditional stability of the inverse problem solution for the geoelectric equation.

\section{Statement of the Problems}

The propagation process of electromagnetic waves in a medium is described by Maxwell's equations [11]:

$$
\begin{gathered}
\varepsilon \frac{\partial}{\partial t} E-\operatorname{rot} H+\sigma E+j^{\mathrm{cm}}=0, \\
x_{3} \neq 0, \quad\left(x_{1}, x_{2}, x_{3}\right) \in R^{3}, \\
\mu \frac{\partial}{\partial t} H+\operatorname{rot} E=0, \quad t>0 .
\end{gathered}
$$

Here $E=\left(E_{1}, E_{2}, E_{3}\right)^{*}$ and $H=\left(H_{1}, H_{2}, H_{3}\right)^{*}$ are the electric and magnetic fields intensity vectors; $\varepsilon$ is dielectric permittivity of the medium; $\mu$ is magnetic permeability of the medium; $\sigma$ is conductivity of the medium; $j^{\mathrm{cm}}$ is source of external currents.
Consider geophysical model of the medium consisting of two half spaces: $R_{-}^{3}=\left\{x \in R^{3}, x_{3}<0\right\}$-air; $R_{+}^{3}=\{x \in$ $\left.R^{3}, x_{3}>0\right\}$-earth.

Let the external current source take the following form:

$$
j^{\mathrm{cm}}=(0,1,0)^{*} g\left(x_{1}\right) \delta\left(x_{3}\right) \theta(t),
$$

where $g\left(x_{1}\right)$ is the function which describes the transversal dimension of the source; $\delta\left(x_{3}\right)$ is Dirac delta function; and $\theta(t)$ is Heaviside function.

Setting the external current in the form (4) makes it an instantaneous inclusion current, parallel to the axis $x_{2}$ at time scales of 10-50 ns (nanoseconds).

Using the definition of the curl we get finally from Maxwell's equations

$$
\begin{aligned}
\varepsilon \frac{\partial}{\partial t} E_{1}+\sigma E_{1} & =\frac{\partial}{\partial x_{2}} H_{3}-\frac{\partial}{\partial x_{3}} H_{2}, \\
\varepsilon \frac{\partial}{\partial t} E_{2}+\sigma E_{2} & =-\frac{\partial}{\partial x_{1}} H_{3}+\frac{\partial}{\partial x_{3}} H_{1}+\gamma_{2}, \\
\varepsilon \frac{\partial}{\partial t} E_{3}+\sigma E_{3} & =\frac{\partial}{\partial x_{1}} H_{2}-\frac{\partial}{\partial x_{2}} H_{1}, \\
\mu \frac{\partial}{\partial t} H_{1} & =-\frac{\partial}{\partial x_{2}} E_{3}+\frac{\partial}{\partial x_{3}} E_{2}, \\
\mu \frac{\partial}{\partial t} H_{2} & =-\frac{\partial}{\partial x_{1}} E_{3}-\frac{\partial}{\partial x_{3}} E_{1}, \\
\mu \frac{\partial}{\partial t} H_{3} & =-\frac{\partial}{\partial x_{1}} E_{2}-\frac{\partial}{\partial x_{2}} E_{1} .
\end{aligned}
$$

Assuming that the coefficients of Maxwell's equations do not depend on the variable $x_{2}$ and are of the special choice of the source in the form (4), the system will retain only three nonzero components $E_{2}, H_{1}$, and $H_{3}$ [11]. Excluding the last two components, the final equations are written such that

$$
\begin{gathered}
\varepsilon \frac{\partial^{2}}{\partial t^{2}} E_{2}+\sigma \frac{\partial}{\partial t} E_{2} \\
=\frac{\partial}{\partial x_{1}}\left(\frac{1}{\mu} \frac{\partial}{\partial x_{1}} E_{2}\right)+\frac{\partial}{\partial x_{3}}\left(\frac{1}{\mu} \frac{\partial}{\partial x_{3}} E_{2}\right) \\
+g\left(x_{1}\right) \eta\left(x_{3}\right) \theta^{\prime}(t), \quad x_{3}>0, t>0 \\
\left.E_{2}\right|_{t>0}=0 \\
\left.\left(\frac{1}{\mu} \frac{\partial}{\partial x_{3}} E_{2}\right)\right|_{x_{3}=+0}=\frac{\partial}{\partial t} \varphi_{(2)}\left(x_{3}, t\right) .
\end{gathered}
$$

Particular attention has aggravated conditions (8) and (9). Condition (8) is taken as additional information (the response of the medium).

Condition (9) is unknown, but it is necessary for solving direct and inverse problems in a half space $\left\{x_{3}>0\right\}$ (earth). 
In this situation we proceed as shown in [11], in the half space $\left\{x_{3} \leq 0\right\}$ where $\sigma=0$ we solve the direct problem by the known data $\varepsilon, \mu$ :

$$
\begin{gathered}
\varepsilon \frac{\partial^{2}}{\partial t^{2}} E_{2}=\frac{\partial}{\partial x_{1}}\left(\frac{1}{\mu} \frac{\partial}{\partial x_{1}} E_{2}\right)+\frac{\partial}{\partial x_{3}}\left(\frac{1}{\mu} \frac{\partial}{\partial x_{3}} E_{2}\right) \\
+g\left(x_{1}\right) \eta\left(x_{3}\right) \theta^{\prime}(t), \quad x_{3}<0, t>0 \\
\left.E_{2}\right|_{t<0}=0 \\
\left.E_{2}\right|_{x_{3}=+0}=\varphi_{(1)}\left(x_{1}, t\right) .
\end{gathered}
$$

In the last system we consider known additional information (8) as a boundary condition for solving the direct problem in the area $\left\{x_{3}<0\right\}$ (air). This fact enables us to restrict the numerical solution of the inverse problem for the minimum possible size of the area in the plane $\left\{x_{3}>0\right\}$.

If the coefficients of (10) do not depend on the variable $x_{1}[11]$ then applying the Fourier transform $F_{x_{1}}[\cdot]$ to (10)(12) and similar to (6)-(9), we write the final statement of the problem.

In the air domain $\left\{x_{3}<0\right\}$ we have the following statement of the direct problem:

$$
\begin{gathered}
\varepsilon \widetilde{v}_{t t}=\frac{1}{\mu} \widetilde{v}_{x_{3} x_{3}}-\frac{\lambda^{2}}{\mu} \widetilde{v}+\widetilde{g}_{\lambda} \eta\left(x_{3}\right) \theta^{\prime}(t), \quad x_{3}<0, \\
\left.\widetilde{v}\right|_{t<0}=0,\left.\quad \widetilde{v}_{t}\right|_{t<0}=0, \\
\widetilde{v}(0, t)=f_{(1)}(t) .
\end{gathered}
$$

In the earth domain $\left\{x_{3}>0\right\}$ we have the following statement of the direct problem:

$$
\begin{gathered}
\widetilde{v}_{t t}+\frac{\sigma}{\varepsilon} \widetilde{v}_{t}=\frac{1}{\mu \varepsilon} \widetilde{v}_{x_{3} x_{3}}-\frac{\lambda^{2}}{\mu \varepsilon} \widetilde{g}_{\lambda} \delta\left(x_{3}, t\right), \quad x_{3}>0, x_{3} \in R^{1} \\
\widetilde{v}_{t<0}=0,\left.\quad \widetilde{v}_{t}\right|_{t<0}=0 \\
\frac{1}{\mu} \widetilde{v}_{x_{3}}(0, t)=f_{(2)}(t) \\
\widetilde{v}(0, t)=f_{(1)}(t)
\end{gathered}
$$

Here $\lambda$ is a Fourier parameter and $\widetilde{v}(x, t)=F_{x_{1}}\left[E_{2}\left(x_{1}\right.\right.$, $\left.\left.0, x_{3}, t\right)\right] ; f_{(1)}(t)=F_{x_{1}}\left[\varphi_{(1)}\left(x_{1}, t\right)\right] ;$ and $f_{(2)}(t)=F_{x_{1}}[(\partial /$ $\left.\partial t) \varphi_{(2)}\left(x_{1}, t\right)\right]$ are Fourier images.

Direct Problem. By the known values of $\varepsilon, \mu$, and $\sigma$ find $\widetilde{v}\left(x_{3}, t\right)$ as the solution of the mixed problem (14)-(16).

Inverse Problem. Find $\sigma\left(x_{3}\right)$ and $\widetilde{v}\left(x_{3}, t\right)$ from (14) to (16) for given $f_{(1)}(t)$ with fixed $\lambda=\lambda_{0}$.

To study conditional stability of the inverse geoelectric problem, it is convenient to use the integral formulation.
Now we introduce the following notations: $b\left(x_{3}\right)=$ $1 / \mu \varepsilon\left(x_{3}\right)$ and $a\left(x_{3}\right)=\sigma\left(x_{3}\right) / \varepsilon\left(x_{3}\right)$ and change the variables and functions:

$$
\begin{gathered}
z=z\left(x_{3}\right)=\int_{0}^{x_{3}} \sqrt{\mu \varepsilon(\xi)} d \xi, \quad x_{3}=\omega(z) ; \\
a(z)=\frac{\sigma(\omega(z))}{\varepsilon(\omega(z))}, \quad b(z)=\frac{1}{\mu \varepsilon(\omega(z))}, \\
u(z, t)=v(\omega(z), t), \quad j_{0}=-g(\lambda) \sqrt{\frac{\mu}{\varepsilon(0)}} .
\end{gathered}
$$

Then (14)-(16) can be written in the form

$$
\begin{gathered}
u_{t t}(z, t)=u_{z z}(z, t)-a(z) u_{t}(z, t) \\
-\frac{b^{\prime}(z)}{b(z)} u_{z}(z, t)-(\lambda b(z))^{2} u(z, t), \\
\left.u\right|_{t<0}=0,\left.\quad u_{t}\right|_{t<0}=0, \\
\frac{1}{\mu} u_{z}(0, t)=f_{(2)}(t), \\
u(0, t)=f_{(1)}(t) .
\end{gathered}
$$

In the future, we will get (19) without the derivative $u_{z}$; for this we assume that

$$
u(z, t)=G(z) v(z, t) .
$$

Now we calculate derivatives as follows:

$$
\begin{gathered}
u_{z}=G^{\prime} v+G v_{z}, \\
u_{z z}=G^{\prime \prime} v+2 G^{\prime} v_{z}+G v_{z z}, \\
u_{t}=G v_{t}, \quad u_{t t}=G v_{t t} .
\end{gathered}
$$

Substituting (24) into (19), we obtain

$$
\begin{aligned}
G v_{t t}= & G^{\prime \prime} v+2 G^{\prime} v_{z}+G v_{z z}-a(z) G v_{t} \\
& -\frac{b^{\prime}}{b}\left(G^{\prime} v+G v_{z}\right)-(\lambda b)^{2} G v .
\end{aligned}
$$

Grouped together, we obtain

$$
\begin{aligned}
v_{t t}= & v_{z z}+\left(2 \frac{G^{\prime}}{G}-\frac{b^{\prime}}{b}\right) v_{z} \\
& -a(z) v_{t}+\left(\frac{G^{\prime \prime}}{G}-\frac{b^{\prime}}{b} \frac{G^{\prime}}{G}-(\lambda b)^{2}\right) v .
\end{aligned}
$$

Put that

$$
\begin{gathered}
2 \frac{G^{\prime}}{G}-\frac{b^{\prime}}{b}=0, \\
g(z)=\frac{G^{\prime \prime}}{G}-\frac{b^{\prime}}{b} \frac{G^{\prime}}{G}-(\lambda b)^{2} .
\end{gathered}
$$


Finally, we have

$$
\begin{gathered}
v_{t t}=v_{z z}-a(z) v_{t}+g(z) v, \\
\left.v\right|_{t<0}=0,\left.\quad v_{t}\right|_{t<0}=0, \\
\frac{1}{\mu} v_{z}(0, t)=f_{(2)}(t), \\
v(0, t)=f_{(1)}(t) .
\end{gathered}
$$

From (27) we have

$$
\begin{gathered}
\frac{G^{\prime}}{G}=\frac{b^{\prime}}{2 b} ; \quad(\ln G)^{\prime}=(\ln \sqrt{b})^{\prime}, \\
\ln G=\ln \sqrt{b}+\ln S(0), \quad S(0)=1, \\
G(z)=\sqrt{b(z)} .
\end{gathered}
$$

Thus, the function $g(z)$ is uniquely determined from (28) by the formula (30).

\section{Statement of the Problem with the Data on the Characteristics}

In the domain $\Delta(l)=\{(z, t)|0<| z \mid<t<l\}$ we consider the inverse problem with data on the characteristics [11]:

$$
\begin{aligned}
v_{t t}(z, t)= & v_{z z}(z, t)-P v(z, t), \quad(z, t) \in \Delta l, \\
& v(z, z)=S(z), \quad 0 \leq z \leq l \\
& v(0, t)=f(t), \quad 0 \leq t \leq 2 l \\
& v_{z}(0, t)=\varphi(t), \quad 0 \leq t \leq 2 l .
\end{aligned}
$$

Here

$$
\begin{gathered}
P v(z, t)=a(z) v_{t}(z, t)+g(z) v(z, t), \\
f(t)=f_{(1)}(t), \quad \varphi(t)=\mu f_{(2)}(t) .
\end{gathered}
$$

We deem that $a(z)$ is an unknown function and the function $g(z)$ is to be known.

Function $S(z)$ is a solution to Volterra integral equation of the second kind:

$$
S(z)=\frac{1}{2} \gamma_{0}-\frac{1}{2} \int_{0}^{z} a(\xi) S(\xi) d \xi, \quad z \in(0, l) .
$$

Inverting the operator $\left(\partial^{2} / \partial t^{2}\right)-\left(\partial^{2} / \partial z^{2}\right)$ in (31) and taking into account (33) and (34), we obtain

$$
v(z, t)=\Phi(z, t)+A_{t, z}[P v], \quad(z, t) \in \Delta(l) .
$$

Here we use the following notations:

$$
\begin{aligned}
& \Phi(z, t)=\frac{1}{2}[f(t+z)+f(t-z)]+\frac{1}{2} \int_{t-z}^{t+z} \varphi(\xi) d \xi \\
& A_{t, z}[v]=\frac{1}{2} \int_{0}^{z} \int_{t-z+\xi}^{t+z-\xi} v(\xi, \tau) d \tau d \xi
\end{aligned}
$$

Differentiating (37) with respect to $t$ we obtain

$v_{t}(z, t)$

$$
=\Phi_{t}(z, t)+\frac{1}{2} \int_{0}^{z}[P v(\xi, t+z-\xi)-P v(\xi, t-z+\xi)] d \xi .
$$

Put $t=z+0$ in (37) and use condition (32); then we have

$$
S(z)=\Phi(z, z+0)+A_{z+0, z}[P v]
$$

Differentiating both sides of the resulting equality with respect to $z$ gives

$$
S^{\prime}(z)=\Phi^{\prime}(z, z+0)+\int_{0}^{z} P v(\xi, 2 z-\xi) d \xi
$$

It is not difficult to see that the function $q(z)=[S(z)]^{-1}$ satisfies Volterra integral equation of the second kind:

$$
q(z)=\gamma^{-1}+\frac{1}{2} \int_{0}^{z} a(\xi) q(\xi) d \xi, \quad \gamma=\frac{\gamma_{0}}{2} .
$$

Taking this into account and the relation $a(z)=$ $2 S^{\prime}(z) / S(z)$, we get

$$
\begin{aligned}
a(z)= & 2\left[\Phi^{\prime}(z, z+0)+\int_{0}^{z} P v(\xi, 2 z-\xi) d \xi\right] \\
& \cdot\left[\gamma^{-1}+\frac{1}{2} \int_{0}^{z} a(\xi) q(\xi) d \xi\right] .
\end{aligned}
$$

Thus, we obtain a closed system of integral equations (37), (39), (42), and (43).

We write this system in vector form as follows:

$$
\Upsilon=F+K(\Upsilon)
$$

where

$$
\begin{gathered}
\Upsilon(z, t)=\left(\Upsilon_{1}, \Upsilon_{2}, \Upsilon_{3}, \Upsilon_{4}\right)^{T}, \\
F(z, t)=\left(F_{1}, F_{2}, F_{3}, F_{4}\right)^{T}, \\
K(\Upsilon)=\left(K_{1}(\Upsilon), K_{2}(\Upsilon), K_{3}(\Upsilon), K_{4}(\Upsilon)\right)^{T}, \\
\Upsilon_{1}(z, t)=v(z, t), \quad \Upsilon_{2}(z, t)=v_{t}(z, t), \\
\Upsilon_{3}(z)=q(z), \quad \Upsilon_{4}(z)=a(z) . \\
F_{1}(z, t)=\Phi(z, t), \quad F_{2}(z, t)=\Phi_{t}(z, t), \\
F_{3}=\gamma_{0}^{-1}, \quad F_{4}(z)=\chi(z),
\end{gathered}
$$


where $\chi(z)=2 \gamma^{-1} \Phi^{\prime}(z, z+0)$,

$$
\begin{aligned}
K_{1}(\Upsilon)= & \frac{1}{2} \int_{0}^{z} \int_{t-z+\xi}^{t+z-\xi} P \Upsilon(\xi, \tau) d \tau d \xi, \quad(z, t) \in \Delta(l), \\
K_{2}(\Upsilon)= & \frac{1}{2} \int_{0}^{z}[P \Upsilon(\xi, t+z-\xi)-P \Upsilon(\xi, t-z+\xi)] d \xi \\
K_{3}(\Upsilon)= & \frac{1}{2} \int_{0}^{z} \Upsilon_{4}(\xi) \Upsilon_{3}(\xi) d \xi \\
K_{4}(\Upsilon)= & \Phi^{\prime}(z, z+0) \int_{0}^{z} \Upsilon_{4}(\xi) \Upsilon_{3}(\xi) d \xi \\
& +2 \int_{0}^{z} P \Upsilon(\xi, 2 z-\xi) d \xi \\
& \cdot\left(\gamma^{-1}+\frac{1}{2} \int_{0}^{z} \Upsilon_{4}(\xi) \Upsilon_{3}(\xi) d \xi\right) .
\end{aligned}
$$

Here

$$
P \Upsilon(z, t)=\Upsilon_{4}(z) \cdot \Upsilon_{2}(z, t)-g(z) \Upsilon_{1}(z, t) .
$$

We deem that $\Upsilon=\left(\Upsilon_{1}, \Upsilon_{2}, \Upsilon_{3}, \Upsilon_{4}\right) \in L_{2}(l)$, if

$$
\begin{gathered}
\Upsilon_{j}(z, t) \in L_{2}(\Delta(l)), \quad j=1,2 ; \\
\Upsilon_{j}(z) \in L_{2}(0, l), \quad j=3,4 .
\end{gathered}
$$

Let $\Upsilon^{(j)}=\left(\Upsilon_{1}^{(j)}(z, t), \Upsilon_{2}^{(j)}(z, t), \Upsilon_{3}^{(j)}(z), \Upsilon_{4}^{(j)}(z)\right)^{T}, j=1,2$. We define the scalar product and the norm as follows:

$$
\begin{aligned}
& \left\langle\Upsilon^{(1)}, \Upsilon^{(2)}\right\rangle \\
& =\sum_{k=1}^{2} \int_{0}^{l} \int_{z}^{2 l-z} \Upsilon_{k}^{(1)}(z, t) \Upsilon_{k}^{(2)}(z, t) d t d z \\
& +\sum_{k=3}^{4} \int_{0}^{l} \Upsilon_{k}^{(1)}(z) \Upsilon_{k}^{(2)}(z) d z, \\
& \|\Upsilon\|^{2}=\langle\Upsilon, \Upsilon\rangle .
\end{aligned}
$$

Inverse Problem. Find vector $\Upsilon \in L_{2}(l)$ from (44) for given $F \in L_{2}(l)$.

\section{Conditional Stability}

Studying $H_{1}$, conditional stability is similar to that in [12] where it was done for the inverse acoustic problem.

We suppose $\|a\|_{L_{2}(0, l)}^{2}=M_{1},\|f\|_{L_{2}(0, l)}^{2}+\left\|f^{\prime}\right\|_{L_{2}(0, l)}^{2}=M_{2}$, $\|\varphi\|_{L_{2}(0, l)}^{2}=M_{3}$, and $\|g\|_{L_{2}(0, l)}^{2} \leq M_{4}$ to be known.

We define $\sum\left(l, M_{1}, a_{*}\right)$ as the class of possible solutions of the inverse problem; namely, $a(z) \in \sum\left(l, M_{1}, a_{*}\right)$ if $a(z)$ satisfies the following conditions:

(1) $a(z) \in H_{1}(0, l) \cap C^{1}(0, l)$,

(2) $\|a\|_{H_{1(0,1)}} \leq M_{1}$,

(3) $0<a_{*} \leq a(z), x \in(0, l)$.
We also define $F\left(l, M_{2}, M_{3}, M_{4}, k_{0}\right)$ as the class of possible initial data; namely, $f \in F\left(l, Q, k_{0}\right)$ if $f$ satisfies the following conditions:

(1) $f \in H_{1}(0,2 l)$,

(2) $\|f\|_{H_{1}(0,2 l)} \leq M_{2}$,

(3) $f(+0)=k_{0},\|\varphi\|_{H_{1}(0,2 l)} \leq M_{3}$.

Suppose that for $f^{(1)}, f^{(2)} \in F\left(l, M_{2}, M_{3}, M_{4}, k_{0}\right)$ there exist $a^{(1)}$ and $a^{(2)}$ from $\sum\left(l, M_{1}, a_{*}\right)$ which solve the inverse problem:

$$
\begin{gathered}
v_{t t}^{(j)}(z, t)=v_{z z}^{(j)}(z, t)-P v^{(j)}(z, t), \quad(z, t) \in \Delta(l), \\
v^{(j)}(z, z)=S^{(j)}(z), \quad 0 \leq z \leq l, \\
v^{(j)}(0, t)=f^{(j)}(t), \quad v_{z}(0, t)=\varphi^{(j)}(t), \quad 0 \leq t \leq 2 l,
\end{gathered}
$$

for $j=1,2$, respectively.

Here

$$
\begin{gathered}
P v^{(j)}(z, t)=a^{(j)}(z) v_{t}^{(j)}(z, t)+g(z) v^{(j)}(z, t), \\
f^{(j)}(t)=f_{(1)}^{(j)}(t), \quad \varphi^{(j)}(t)=\mu f_{(2)}^{(j)}(t) .
\end{gathered}
$$

We deem that the function $g(z)$ is known and $a^{(j)}(z)$ is unknown, $j=1,2$. We write the early resulting closed system in the vector form as follows:

$$
\Upsilon^{(j)}=F^{(j)}+K\left(\Upsilon^{(j)}\right), \quad j=1,2,
$$

where

$$
\begin{gathered}
\Upsilon^{(j)}=\left(\Upsilon_{1}^{(j)}, \Upsilon_{2}^{(j)}, \Upsilon_{3}^{(j)}, \Upsilon_{4}^{(j)}\right)^{T} ; \\
\Upsilon_{1}^{(j)}(z, t)=v^{(j)}(z, t), \quad \Upsilon_{2}^{(j)}(z, t)=v_{t}^{(j)}(z, t), \\
\Upsilon_{3}^{(j)}(z)=q(z), \quad \Upsilon_{4}^{(j)}(z)=a^{(j)}(z) ; \\
F^{(j)}=\left(F_{1}^{(j)}, F_{2}^{(j)}, F_{3}^{(j)}, F_{4}^{(j)}\right)^{T}, \quad j=1,2 ; \\
F_{1}^{(j)}(z, t)=\Phi^{(j)}(z, t), \quad F_{2}^{(j)}(z, t)=\Phi_{t}^{(j)}(z, t), \\
F_{3}=j^{-1}, \quad F_{4}(z)=\chi^{(j)}(z), \quad j=1,2 ; \\
K_{1}\left(\Upsilon^{(j)}\right)=\frac{1}{2} \int_{0}^{z} \int_{t-z+\xi}^{t+z-\xi} P \Upsilon^{(j)}(\xi, \tau) d \tau d \xi, \quad(z, l) \in \Delta(l),
\end{gathered}
$$




$$
\begin{aligned}
& K_{2}\left(\Upsilon^{(j)}\right) \\
& =\frac{1}{2} \int_{0}^{z}\left[P \Upsilon^{(j)}(\xi, t+z-\xi)-P \Upsilon^{(j)}(\xi, t-z+\xi)\right] d \xi \\
& K_{3}\left(\Upsilon^{(j)}\right)=\frac{1}{2} \int_{0}^{z} \Upsilon_{4}^{(j)} \Upsilon_{3}^{(j)} d \xi \\
& K_{4}\left(\Upsilon^{(j)}\right)= \\
& \Phi^{\prime(j)}(z, z+0) \int_{0}^{z} \Upsilon_{4}^{(j)}(\xi) \Upsilon_{3}^{(j)}(\xi) d \xi \\
& +2 \int_{0}^{z} P \Upsilon^{(j)}(\xi, 2 z-\xi) d \xi \\
& \cdot\left(\gamma^{-1}+\frac{1}{2} \int_{0}^{z} \Upsilon_{4}^{(j)}(\xi) \Upsilon_{3}^{(j)}(\xi) d \xi\right) \\
& \text { labeleq55 }
\end{aligned}
$$

Here we denote $P \Upsilon^{(j)}(z, t)=\Upsilon_{4}(z) \Upsilon_{2}(z, t)-g(z) \Upsilon_{1}(z, t)$.

Theorem 1. Suppose that, for $F^{(j)} \in L_{2}(l), j=1,2$, there exist $\Upsilon^{(j)} \in L_{2}(\Delta(l))$ as the solution of the inverse problem as follows:

$$
\Upsilon^{(j)}(z, t)=F^{(j)}(z, t)+K\left(\Upsilon^{(j)}\right), \quad j=1,2,(z, t) \in \Delta(l) .
$$

Then

$$
\left\|\Upsilon^{(1)}-\Upsilon^{(2)}\right\|^{2} \leq C\left\|f^{(1)}-f^{(2)}\right\|_{H_{1}(0,2 l)}^{2}
$$

where

$$
C=C\left(l, M_{1}, M_{2}, M_{3}, M_{4}, k_{0}\right) .
$$

Proof. We introduce

$$
\begin{gathered}
\tilde{\Upsilon}(x, t)=\left(\widetilde{\Upsilon}_{1}(z, t), \widetilde{\Upsilon}_{2}(z, t), \widetilde{\Upsilon}_{3}(z), \widetilde{\Upsilon}_{4}(z)\right) \\
=\Upsilon^{(1)}(z, t)-\Upsilon^{(2)}(z, t), \\
\tilde{F}(z, t)=F^{(1)}(z, t)-F^{(2)}(z, t) .
\end{gathered}
$$

Then from (52) it follows that

$$
\widetilde{\Upsilon}(z, t)=\widetilde{F}(z, t)-K(\widetilde{\Upsilon}), \quad(z, t) \in \Delta(l) .
$$

In the vector equation (60) we estimate each component separately taking into account the obvious inequalities as follows:

$$
\begin{gathered}
(a+b+c)^{2} \leq 3\left(a^{2}+b^{2}+c^{2}\right), \\
(\sqrt{a}+\sqrt{b})^{2} \leq 2 a+2 b
\end{gathered}
$$

for $a \geq 0, b \geq 0$.

We obtain the chain of the inequalities:

$$
\begin{aligned}
\left|\widetilde{\Upsilon}_{1}(z, t)\right| \leq\left|\widetilde{F}_{1}(z, t)\right|+\frac{1}{2} \sqrt{\int_{0}^{z}\left|\widetilde{\Upsilon}_{3}(\xi)\right|^{2} d \xi} & \\
\times & {\left[\sqrt{\int_{0}^{z}\left|\Upsilon_{1}^{(1)}(\xi, t+z-\xi)\right|^{2} d \xi}\right.} \\
& \left.+\sqrt{\int_{0}^{z}\left|\Upsilon_{1}^{(1)}(\xi, t-z+\xi)\right|^{2} d \xi}\right] \\
+ & \frac{1}{2} \sqrt{\int_{0}^{z}\left|\Upsilon_{3}^{(2)}(\xi)\right|^{2} d \xi} \\
\times & {\left[\sqrt{\int_{0}^{z}\left|\widetilde{\Upsilon}_{1}(\xi, t+z-\xi)\right|^{2} d \xi}\right.} \\
& \left.+\sqrt{\int_{0}^{z}\left|\widetilde{\Upsilon}_{2}(\xi, t-z+\xi)\right|^{2} d \xi}\right] .
\end{aligned}
$$

Using the obvious inequality we get

$$
\begin{aligned}
\left|\widetilde{\Upsilon}_{1}(z, t)\right|^{2} & \\
\leq & 3\left|\widetilde{F}_{1}(z, t)\right|^{2}+\frac{3}{2} \int_{0}^{z}\left|\widetilde{\Upsilon}_{3}(\xi)\right|^{2} d \xi \\
& \times \int_{0}^{z}\left[\left|\Upsilon_{1}^{(1)}(\xi, t+z-\xi)\right|^{2}+\left|\Upsilon_{1}^{(1)}(\xi, t-z+\xi)\right|^{2}\right] d \xi \\
& +\frac{3}{2} \int_{0}^{z}\left|\Upsilon_{3}^{(2)}(\xi)\right|^{2} d \xi \\
& \times \int_{0}^{z}\left|\widetilde{\Upsilon}_{1}(\xi, t+z-\xi)\right|^{2}+\left|\widetilde{\Upsilon}_{1}^{(2)}(\xi, t-z+\xi)\right|^{2} d \xi
\end{aligned}
$$

Turning to the earlier introduced norms we have

$$
\begin{aligned}
& \left\|\widetilde{\Upsilon}_{1}\right\|_{L_{2}(\Delta(l, z))}^{2} \\
& \leq 3\left\|\widetilde{F}_{1}\right\|_{L_{2}(\Delta(l, z))^{T}}^{2} \\
& +\frac{3}{2} \int_{0}^{z} \int_{\xi}^{2 l-\xi}\left\{\int_{0}^{\xi}\left|\widetilde{\Upsilon}_{3}\left(\xi^{\prime}\right)\right|^{2} d \xi^{\prime}\right. \\
& \quad \times \int_{0}^{\xi}\left[\left|\Upsilon_{1}^{(1)}\left(\xi^{\prime}, \tau+\xi-\xi^{\prime}\right)\right|^{2}\right.
\end{aligned}
$$




$$
\begin{gathered}
\left.+\left|\Upsilon_{1}^{(1)}\left(\xi^{\prime}, \tau-\xi+\xi^{\prime}\right)\right|^{2}\right] d \xi^{\prime} \\
+\int_{0}^{\xi}\left|\Upsilon_{3}^{2}\left(\xi^{\prime}\right)\right|^{2} d \xi^{\prime} \\
\times \int_{0}^{\xi}\left[\left|\widetilde{\Upsilon}_{1}\left(\xi^{\prime}, \tau+\xi-\xi^{\prime}\right)\right|^{2}\right. \\
\left.\left.+\left|\widetilde{\Upsilon}_{1}^{(2)}\left(\xi^{\prime}, \tau-\xi+\xi^{\prime}\right)\right|^{2}\right] d \xi^{\prime}\right\} d \tau d \xi
\end{gathered}
$$

$\leq 3\left\|\widetilde{F}_{1}\right\|_{L_{2}(\Delta(l, z))}^{2}$

$$
+12 \Upsilon_{*}^{2} \int_{0}^{z} \int_{0}^{\xi}\left|\widetilde{\Upsilon}_{3}\left(\xi^{\prime}\right)\right|^{2} d \xi^{\prime} d \xi+12 \Upsilon_{*}^{2} \int_{0}^{z}\left\|\widetilde{\Upsilon}_{1}\right\|_{L_{2}(\Delta(l, \xi))} d \xi
$$

Here

$$
\Upsilon_{*}=\max \left\{\left\|\Upsilon^{(1)}\right\|,\left\|\Upsilon^{(2)}\right\|\right\}
$$

We estimate the second component of (60):

$$
\begin{aligned}
\left|\widetilde{\Upsilon}_{2}(z)\right| \leq & \frac{1}{2} \int_{0}^{z}\left|\Upsilon_{3}^{(1)}(\xi) \tilde{\Upsilon}_{2}(\xi)\right| d \xi \\
& +\frac{1}{2} \int_{0}^{z}\left|\Upsilon_{2}^{(2)}(\xi) \tilde{\Upsilon}_{3}(\xi)\right| d \xi \\
\leq & \frac{1}{2} \sqrt{\int_{0}^{z}\left|\Upsilon_{3}^{(1)}(\xi)\right|^{2} d \xi} \sqrt{\int_{0}^{z}\left|\widetilde{\Upsilon}_{2}(\xi)\right|^{2} d \xi} \\
& +\frac{1}{2} \sqrt{\int_{0}^{z}\left|\Upsilon_{2}^{(2)}(\xi)\right|^{2} d \xi} \sqrt{\int_{0}^{z}\left|\widetilde{\Upsilon}_{3}(\xi)\right|^{2} d \xi}
\end{aligned}
$$

Then we have

$$
\left\|\widetilde{\Upsilon}_{2}\right\|_{L_{2}(0, z)}^{2} \leq \frac{1}{2} \Upsilon_{*}^{2} \int_{0}^{z}\left[\left\|\widetilde{\Upsilon}_{2}\right\|_{L_{2}(0, \xi)}^{2}+\left\|\widetilde{\Upsilon}_{3}\right\|_{L_{2}(0, \xi)}^{2}\right] d \xi
$$

We estimate the third component of (60) and we have

$$
\left\|\widetilde{\Upsilon}_{3}\right\|_{L_{2}(0, l)}^{2} \leq \frac{1}{4} M_{2} \int_{0}^{z}\left[\left\|\widetilde{\Upsilon}_{3}\right\|_{L_{2}(0, \xi)}^{2}+M_{3}\left\|\widetilde{\Upsilon}_{4}\right\|_{L_{2}(0, \xi)}^{2}\right] d \xi
$$

Finally, for the fourth component of (60) we get the estimate

$$
\begin{aligned}
& \left|\widetilde{\Upsilon}_{4}(z)\right| \leq\left|\widetilde{F}_{4}(z)\right|+\sum_{i=1}^{4} w_{i}, \\
& w_{1}(z)=2\left|\left(f^{(1)}\right)^{\prime}(z)\right|\left|K_{2}\left(\Upsilon^{(1)}\right)-K_{2}\left(\Upsilon^{(2)}\right)\right|, \\
& w_{2}(z)=\left|K_{6}\left(\Upsilon^{(1)}\right)-K_{6}\left(\Upsilon^{(2)}\right)\right|,
\end{aligned}
$$

$$
\begin{aligned}
w_{3} & =\left|K_{2}\left(\Upsilon^{(1)}\right)\right| w_{2}(z), \\
w_{4} & =\left|K_{4}\left(\Upsilon^{(2)}\right)\right|\left|K_{2}\left(\Upsilon^{(1)}\right)-K_{2}\left(\Upsilon^{(2)}\right)\right|, \\
w_{5} & =\left|\left(f^{(1)}\right)^{\prime}-\left(f^{(2)}\right)\right|\left|K_{2}\left(\Upsilon^{(2)}\right)\right|, \\
K_{6}(\Upsilon) & =\int_{0}^{z} \Upsilon_{3}(\xi) \Phi_{1}(\xi, 2 z-\xi) d \xi .
\end{aligned}
$$

Estimating each term $w_{i}(z)$ and substituting into (69) and using the obvious inequality

$$
\left(\sum_{k=1}^{4}\left|b_{k}\right|\right)^{2} \leq 4 \sum_{k=1}^{4}\left|b_{k}\right|^{2}
$$

we obtain

$$
\begin{aligned}
& \left\|\widetilde{\Upsilon}_{4}(z)\right\|_{L_{2}(0, z)} \\
& \leq v_{0} \int_{0}^{z}\left[f^{\prime}(2 \xi)\right]^{2} d \xi+\frac{1}{2} v_{1}\left\|\widetilde{\Upsilon}_{1}\right\|_{L_{2}(\Delta(l, z))} \\
& \quad+\int_{0}^{z} v_{3}(\xi)\left\|\widetilde{\Upsilon}_{2}\right\|_{L_{2}(0, \xi)} d \xi \\
& \quad+\int_{0}^{z} v_{3}(\xi)\left\|\widetilde{\Upsilon}_{3}\right\|_{L_{2}(0, \xi)} d \xi \\
& \quad+\int_{0}^{z} v_{4}(\xi)\left\|\widetilde{\Upsilon}_{4}\right\|_{L_{2}(0, \xi)} d \xi .
\end{aligned}
$$

Now we combine all the obtained estimates for the four components (60) and denote, for convenience,

$$
\psi_{1}(z)=\left\|\widetilde{\Upsilon}_{1}\right\|_{L_{2}(\Delta(l, z))}^{2}, \quad z \in(0, l)
$$

and then

$$
\psi(z)=\psi_{1}(z)+\psi_{2}(z)+\psi_{3}(z)+\psi_{4}(z)
$$

and for function $\psi$ we obtain the following estimate:

$$
\psi(z) \leq \eta+\int_{0}^{z} \sum_{i=1}^{4} \gamma_{i}(\xi) \psi_{i}(\xi) d \xi
$$

where $\eta=\eta\left(\Upsilon_{*}^{2}, v_{1}, v_{2}, v_{3}, v_{4}\right)$.

Introduce a new function:

$$
\nu(z)=\eta_{*}+\int_{0}^{z} \sum_{i=1}^{4} \gamma_{i}(\xi) \psi_{i}(\xi) d \xi, \quad \eta<\eta_{*},
$$

where $\eta_{*}$ is constant.

Then $\psi(z) \leq v(z)$,

$$
\begin{gathered}
\nu^{\prime}(z)=\sum_{i=1}^{4} \gamma_{i}(z) \Upsilon_{j}(z) \leq \nu(z) \sum_{i=1}^{4} \gamma_{i}(z), \\
\frac{\nu^{\prime}(z)}{\nu(z)} \leq \sum_{i=1}^{4} \gamma_{i}(z) .
\end{gathered}
$$


Applying the Gronwall inequality we obtain

$$
\begin{gathered}
\psi(z) \leq \nu(z) \leq v(0) \exp \left\{\int_{0}^{z} \sum_{i=1}^{4} \gamma_{i}(\xi) d \xi\right\}, \\
\int_{0}^{z} \sum_{i=1}^{4} \gamma_{i}(\xi) d \xi \leq 25 \Upsilon_{*}^{2} \times z+12 \Upsilon_{*}^{2}\left\|f^{(1)}\right\|_{L_{2}(0,2 l)}^{2} \\
+12 \Upsilon_{*}^{4}+12 \Upsilon_{*}^{2}\left(12+\Upsilon_{*}^{2} \cdot z\right) .
\end{gathered}
$$

Then from (77) we obtain

$$
\left\|\Upsilon^{(1)}-\Upsilon^{(2)}\right\|^{2} \leq \widetilde{N}\left\|f^{(1)}-f^{(2)}\right\|_{H_{1}(0,2 l)}
$$

where the constant $C>0$ is given by (58).

An explicit expression for the constant as a result of successive computations is given by

$$
\begin{gathered}
C=\left[6 l+6 M_{1}\left(\frac{4}{k_{0}^{2}}+\Upsilon_{*}^{4}\right)\left(1+12 \Upsilon_{*}^{2} l\right)\right] \\
\times \exp \left\{\Upsilon _ { * } ^ { 2 } \left[24 l+8 M_{2}\left(\frac{4}{k_{0}^{2}}+\Upsilon_{*}^{4}\right)\left(M_{3}+36 \Upsilon_{*}^{2} l\right)\right.\right. \\
\left.\left.+6 M_{2} \Phi^{2}+8 M_{4} \Upsilon_{*}^{4}\right]\right\} .
\end{gathered}
$$

\section{Conclusions}

The conditional stability of the inverse problem for the geoelectric equation has been investigated. For studying we consider the integral formulation of the inverse geoelectric problem. The estimation of the conditional stability of the inverse problem solution has been obtained or rather lower changes in input data imply lower changes in the solution (of the numerical method). When determining the additional information the device errors are possible. That is why this research is important for experimental studies with usage of ground penetrating radars. The inlet data belongs to the class $F\left(l, M_{2}, M_{3}, M_{4}, k_{0}\right)$, while the solution belongs to the class $\sum\left(l, M_{1}, a_{*}\right)$.

\section{Conflict of Interests}

The authors declare that there is no conflict of interests regarding the publication of this paper.

\section{Acknowledgment}

The work was supported by Ministry of Education and Science of the Republic of Kazakhstan (Grant no. 139 (69) от 04.02.2014).

\section{References}

[1] S. I. Kabanikhin, Inverse and III-Posed Problems: Theory and Applications, de Gruyter, Berlin, Germany, 2011.
[2] A. S. Blagoveshchenskii and V. M. Babič, "On a local method of solution of a nonstationary inverse problem for a nonhomogeneous string," in Mathematical Questions in the Theory of Wave Diffraction, vol. 115 of Proceedings of the Steklov Institute of Mathematics in the Academy of Sciences of the USSR, pp. 30-41, American Mathematical Society, Providence, RI, USA, 1974.

[3] V. G. Romanov, Inverse Problems of Mathematical Physics, VNU Science Press, Utrecht, The Netherlands, 1987.

[4] S. I. Kabanikhin and A. Lorenzi, Identification Problems of Wave phenomena, VSP, Utrecht, The Netherlands, 1999.

[5] V. G. Romanov and M. Yamamoto, "Multidimensional inverse hyperbolic problem with impulse input and a single boundary measurement," Journal of Inverse and Ill-Posed Problems, vol. 7, no. 6, pp. 573-588, 1999.

[6] V. Baranov and G. Kunetz, "Synthetic seismograms with multiplenreflections: theory and numerical experience," Geophysical Prospecting, vol. 8, pp. 315-325, 1969.

[7] A. Bamberger, G. Chavent, C. Hemon, and P. Lailly, "Inversion of normal incidence seismograms," Geophysics, vol. 47, no. 5, pp. 757-770, 1982.

[8] A. Bamberger, G. Chavent, and P. Lailly, "About the stability of the inverse problem in 1-D wave equations-applications to the interpretation of seismic profiles," Applied Mathematics and Optimization, vol. 5, no. 1, pp. 1-47, 1979.

[9] S. He and S. I. Kabanikhin, "An optimization approach to a three-dimensional acoustic inverse problem in the time domain," Journal of Mathematical Physics, vol. 36, no. 8, pp. 4028-4043, 1995.

[10] J. S. Azamatov and S. I. Kabanikhin, "Volterra operator equations. $L_{2}$-theory," Journal of Inverse and Ill-Posed Problems, vol. 7, no. 6, pp. 487-510, 1999.

[11] V. G. Romanov and S. I. Kabanikhin, Inverse Problems for Maxwell's Equations, VSP, Utrecht, The Netherlands, 1994.

[12] S. I. Kabanikhin, K. T. Iskakov, and M. Yamamoto, " $\mathrm{H}^{1}$ conditional stability with explicit Lipshitz constant for a onedimensional inverse acoustic problem," Journal of Inverse and Ill-Posed Problems, vol. 9, no. 3, pp. 249-267, 2001.

[13] S. I. Kabanikhin and K. T. Iskakov, "Justification of the steepest descent method in an integral formulation of an inverse problem for a hyperbolic equation," Siberian Mathematical journal, vol. 42, no. 3, pp. 478-494, 2001.

[14] S. I. Kabanikhin and K. T. Iskakov, Optimization Methods of Coefficient Inverse Problems Solution, NGU, Novosibirsk, Russia, 2001.

[15] A. L. Bukhgeim and M. V. Klibanov, "Uniqueness in the large of a class of multidimensional inverse problems," Soviet Mathematics Doklady, vol. 17, pp. 244-247, 1981.

[16] M. V. Klibanov, "Carleman estimates for global uniqueness, stability and numerical methods for coefficient inverse problems," Journal of Inverse and Ill-Posed Problems, vol. 21, no. 4, pp. 477560, 2013.

[17] M. V. Klibanov, "Uniqueness of the solution of two inverse problems for a Maxwell system," Computational Mathematics and Mathematical Physics, vol. 26, no. 7, pp. 67-73, 1986.

[18] A. V. Kuzhuget, L. Beilina, M. V. Klibanov, A. Sullivan, L. Nguyen, and M. A. Fiddy, "Blind backscattering experimental data collected in the field and an approximately globally convergent inverse algorithm," Inverse Problems, vol. 28, no. 9, Article ID 095007, 2012.

[19] L. Beilina and M. V. Klibanov, Approximate Global Convergence and Adaptivity for Coefficient Inverse Problems, Springer, New York, NY, USA, 2012. 
[20] A. L. Karchevsky, M. V. Klibanov, L. Nguyen, N. Pantong, and A. Sullivan, "The Krein method and the globally convergent method for experimental data," Applied Numerical Mathematics, vol. 74, pp. 111-127, 2013.

[21] S. I. Kabanikhin, D. B. Nurseitov, M. A. Shishlenin, and B. B. Sholpanbaev, "Inverse problems for the ground penetrating radar," Journal of Inverse and Ill-Posed Problems, vol. 21, no. 6, pp. 885-892, 2013. 


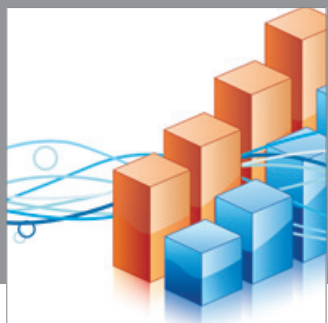

Advances in

Operations Research

mansans

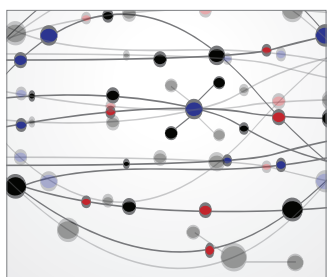

The Scientific World Journal
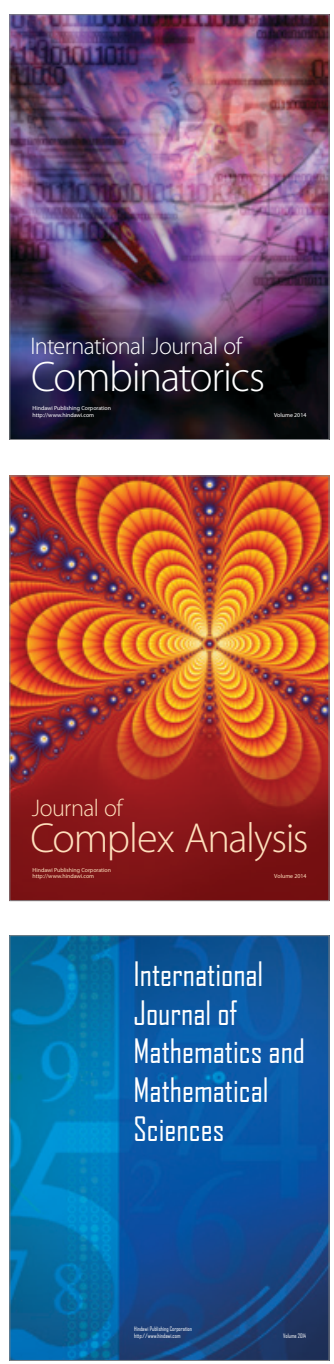
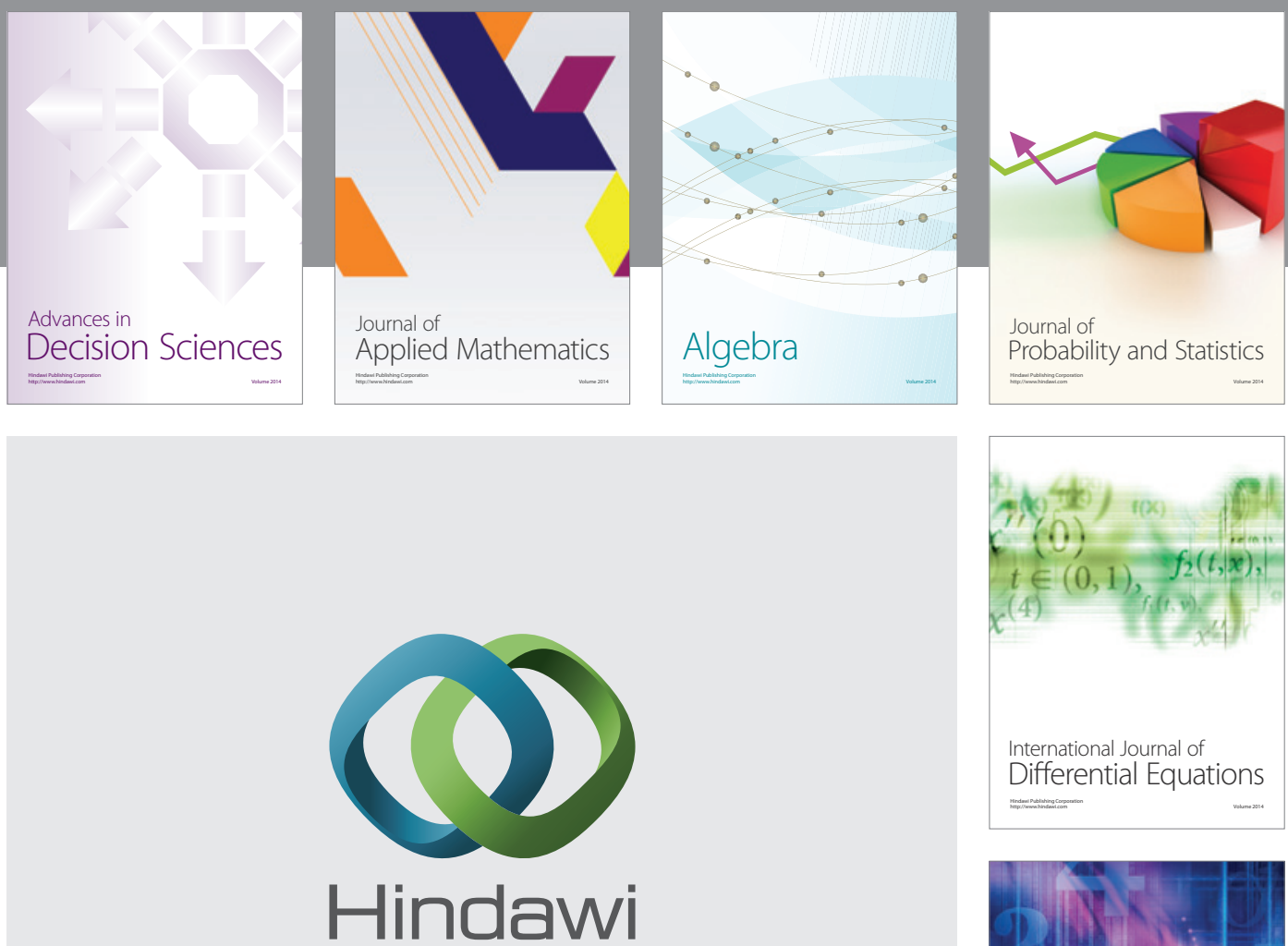

Submit your manuscripts at http://www.hindawi.com
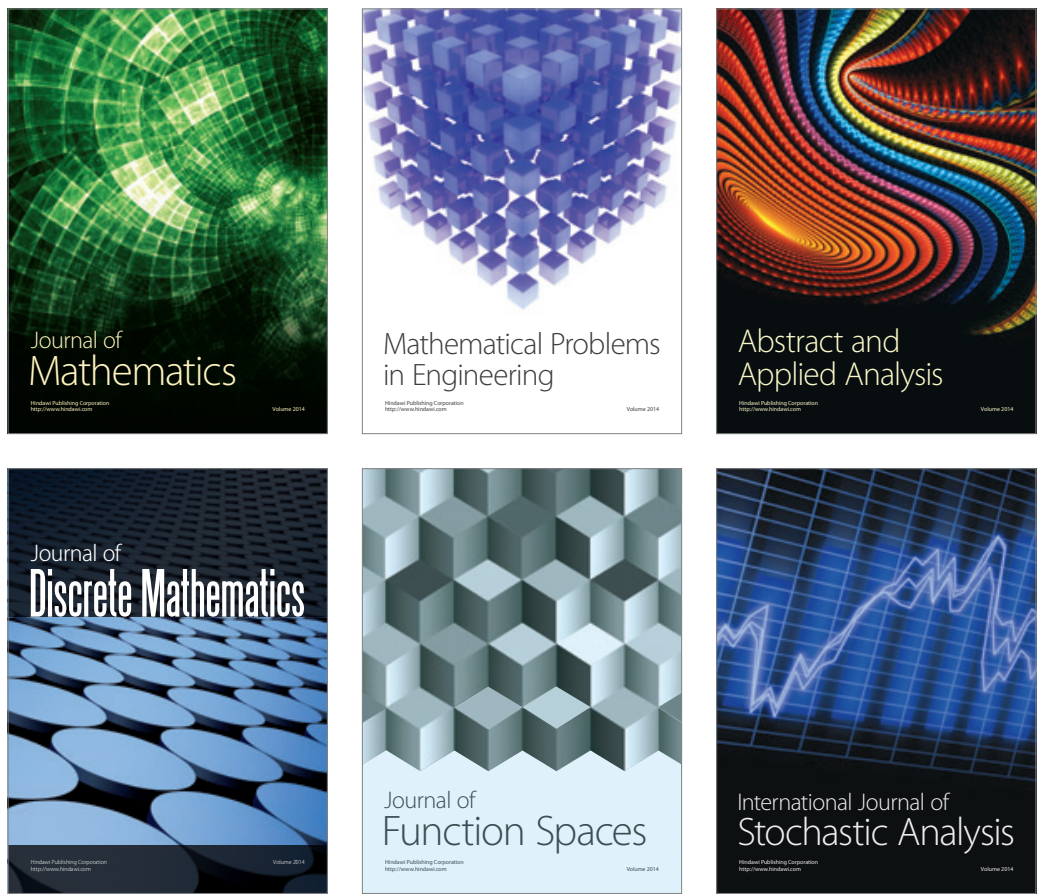

Journal of

Function Spaces

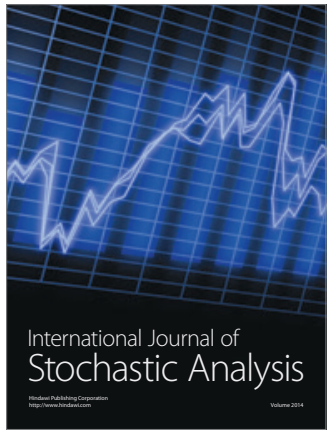

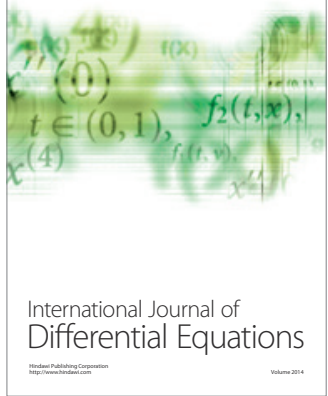
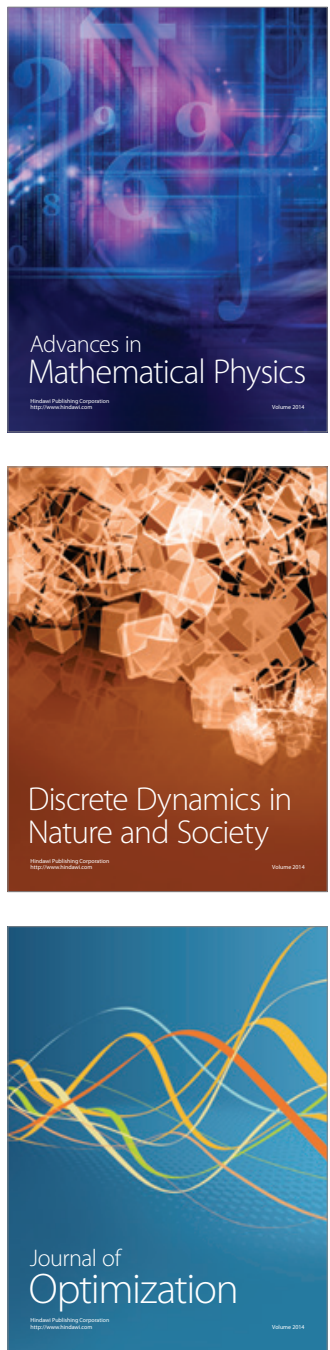